\title{
Validity range of wind turbine models.
}

\author{
M.P. Comech, D. García García, M. Sanz, J. Martínez García, M. García-Gracia \\ CIRCE-Departamento de Ingeniería Eléctrica de la Universidad de Zaragoza \\ C/ María de Luna n 3, 50018 Zaragoza (España) \\ Email: mggracia@posta.unizar.es
}

\begin{abstract}
-
In this paper, the focus is on fundamental frequency simulations, also known as electromechanical transient simulations. In this type of simulation, the network is represented as an impedance matrix and only the fundamental frequency component of voltages and currents is taken into account in order to reduce the computation time. This simulation approach is mainly used for voltage and angle stability investigations. Models of wind turbine generating systems, which match the fundamental frequency simulation approach, are presented and their responses are compared with an electromagnetic transient model.
\end{abstract}

\section{Key words:}

Grid interaction, modelling, power system dynamics, simulation, wind power.

\section{Introduction}

Numbers of wind turbines are increasing, a major problem is to determine their influence on the electrical power systems. The wind turbine impact on the power systems becomes an important subject, studied by means of dynamic simulations.

The power system dynamic is evaluated by simulation, and companies usually use the dynamical module of software package as PSS/E [1], Eurostag,... A large power system can easily have up to thousands of branches, generators and their controllers and, when dynamic load models are used, also with loads. Hence, long simulation runs are required.

To allow the study of the impact of wind energy penetrations on power systems, wind turbine models are developed for software as PSS/E in which the fundamental frequency simulation approach is applied. These models must match the assumptions and simplifications generally made in fundamental frequency simulations.

Many wind turbines models can be found in the literature. However, most of the available models cannot easily be applied for fundamental frequency simulations, because they contain time constants, which cannot be taken into account in fundamental frequency simulations.

\section{PSS/E description}

The dynamic simulation tool PSS/E is commonly applied in study of power system stability of large power systems. PSS/E is a fundamental frequency tool; which implies higher harmonics are not represented in the simulation.

The network model in PSS/E is stablished by the positive-sequence equivalents. The behavior of a system is described by means of a set of differential equations considering state variables. At each time instant, the time derivative of each state variable is calculated, with the constant and variable parameters, which describe the condition of the system at that time instant in detail, as initial conditions. From the obtained value of each state variable and its time derivative, the next state variable values is determined. The process is repeated increasing the simulation time.

The generator models in $\mathrm{PSS} / \mathrm{E}$ are reduced order models, which transients effects of the machine are neglected. Such assumptions are acceptable with balanced events and modelling synchronous generators of large power plants [2].

Therefore, it is necessary the PSS/E model comparison with a more advanced model in other simulation tool which transient studies are allowed, as PSCAD [3].

\section{Wind turbine model}

Several wind turbine generating systems are currently applied.:

- Synchronous generator. It is grid coupled through a back-to-back voltage source converter or a diode rectifier and voltage source converter. The synchronous generator is a low speed multipole generator; then gearbox is unnecessary. For high wind speeds, the power generated is limited by pitch control.

- The doubly-fed induction generator. It also allows variable speed operation. The rotor winding is fed using a back-to-back voltage source converter. In this case the wind turbine rotor is coupled to the generator through a gearbox. For high wind speeds, the power extracted from the wind is also limited by a pitch control.

- Squirrel cage induction generator with constant speed. This device is usually directly grid coupled, The wind turbine rotor is coupled to the generator through a gearbox. In these turbines, the power 
extracted from the wind is limited using the stall effect. So the rotor is designed in such a way that its aerodynamic efficiency decreases for high wind speeds. When the stall effect is used, no active control systems are necessary.

A widely used squirrel cage induction generator model has been considered [4]. The induction machine is modelled using a Thevenin equivalent as it can be seen in Figure 1.

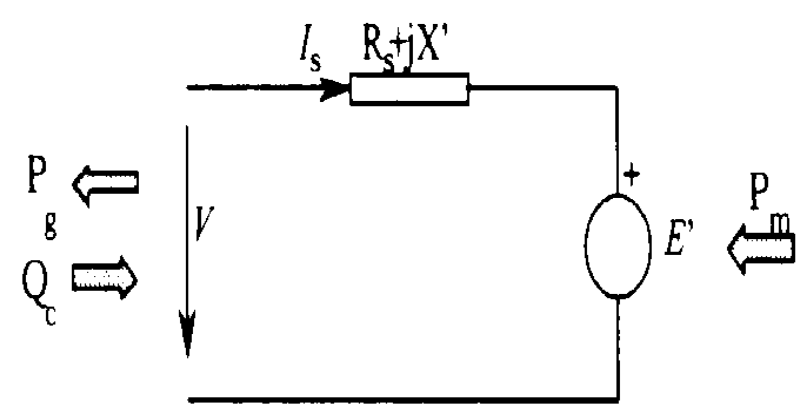

Figure 1. Thevenin equivalent

In this model, $R_{s}$ is the stator resistance, $X^{\prime}$ the transient reactance. The voltage source $E^{\prime}$ is a complex variable which governs by means of the following equation differential:

$$
\frac{d E^{\prime}}{d t}=j 2 \pi f_{0} s \cdot\left(V_{r}^{\prime}-E^{\prime}\right)-\frac{1}{T_{0}{ }^{\prime}}\left[E^{\prime}-j\left(X-X^{\prime}\right) I_{s}\right]
$$

where $f_{0}$ is the electrical frequency of the network (in Hertz), $s$ is the slip of the generator and $I_{s}$ the intensity of the stator (intensity consumed by the generator). $\mathrm{Vr}$ ' is the rotor feeding voltage, whose value is zero in the case of being a machine with the rotor shortcircuited.

The constants of the previous equation are referred to the equivalent circuit of an asynchronous machine in permanent state shown in Figure 2. Next these constants are detailed.

- $\mathrm{X}^{\prime}$ is the transient reactance of the machine (short circuit reactance):

$$
X^{\prime}=X_{s}+\frac{X_{R} \cdot X_{m}}{X_{R}+X_{m}}
$$

- T0 $^{\prime}$ is the transient constant of time at open circuit

$$
T_{0}{ }^{\prime}=\frac{X_{R}+X_{m}}{2 \cdot \pi \cdot f_{0} \cdot R_{R}}
$$

- $\mathrm{X}$ is the generator reactance at open circuit

$$
X=X_{s}+X_{m}
$$

where $X_{m}$ is the magnetizing reactance of the generator and $\mathrm{Rr}$ the resistance of the rotor. $R_{S}, R_{r}, X_{s}, X_{r}$ and $X_{m}$ are obtained with the values of the equivalent circuit of an asynchronous machine (Figure 2).

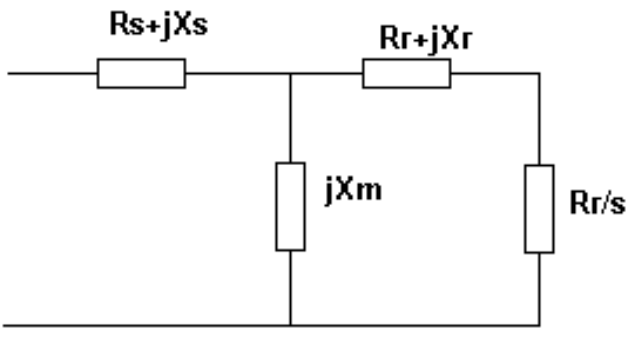

Figure 2. Single-phase equivalent of the asynchronous machine in permanent state.

\section{A. Mechanical Set}

The wind energy is caught by the blades of the wind turbine. The blades are made of composite material (polyester, fiber glass and carbon among others) which allows to obtain a high resistance with a reduced weight. These blades turn at an almost constant speed of approximately 20-30 rpm. As this speed is clearly insufficient to produce electrical energy with a generator, a gearbox is used to elevate the speed of 40 to 100 times the speed of the blades, up to $1500 \mathrm{rpm}$.

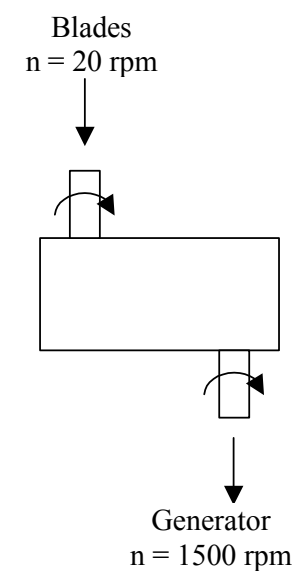

Figure 3. Gearbox layout.

A equivalent moment of inertia is calculated as the sum of the generator, gearbox, hub and blades moments of inertia. The blades represent the major contribution to the sum.

$$
J_{\text {eq }}=J_{\text {gen }}+J_{\text {gearbox }}+J_{\text {hub }}^{\prime}+J_{\text {blades }}^{\prime}
$$

where

$$
\begin{gathered}
J_{\text {blades }}^{\prime}+J_{\text {hub }}^{\prime}=\left(J_{\text {blades }}+J_{\text {hub }}\right) \cdot\left(\frac{1}{r_{r}}\right)^{2} \text { and } \\
r_{r}=\frac{n_{\text {generator }}}{n_{\text {blades }}}
\end{gathered}
$$

The inertia constant $\mathrm{H}$ is the fundamental parameter in stability studies, giving an idea of the electromechanical response time of the machine. In the machine base, $\mathrm{H}$ is defined as: 


$$
H=\frac{\frac{1}{2} \cdot J \cdot\left(\frac{2 \cdot \pi \cdot f_{r e d}}{p}\right)^{2}}{S_{b}}
$$

where

- $H$ is the inertia constant of the machine on seconds (MJ/MVA)

- $J$ is the moment of inertia of the machine referred to the axis of the alternator $\left(\mathrm{Kg} \cdot \mathrm{m}^{2}\right)$.

- $f_{\text {red }}$ is the network frequency in $\mathrm{Hz}$.

- $\quad p$ is the number of pairs of poles of the machine.

- $S_{b}$ is the machine power bases of the on VA.

The ecuation shows that $H$ is the relation between the kinetic energy of the generator at the synchronism speed and the power base of the machine. In wind turbines, the range of the constant of inertia reaches values between 2 and 7 seconds. This value is very high when it is compared to any other conentional power generation $(\mathrm{H}$ varies between 2 and $4 \mathrm{~s}$ ); but this is only apparent, since in the expression of $\mathrm{H}$ is divided between machine power base, which is very low in wind stations.

\section{B. Power Curve of the Wind Turbine}

The wind speed is extremely important for the amount of energy a wind turbine can convert to electricity. The power curve is a main characteristic of the machine which represents the electrical power produced by a wind turbine for any wind speed.

This curve and the wind distribution of the zone allows to calculate the generated energy by the machine.

\section{Harvest energy from the wind}

The amount of energy which the turbine harvest from the wind depends on three factors:

- The wind power available. The value of this power depends on the wind distribution, which is a characteristic of the wind farm location.

- The power curve of the machine which is function of the kind of machine, and

- The good behavior of the machine when it has to respond to fluctuations in the wind velocity. This is a characteristic of the technology used.

The energy from the wind is expressed, of general form with the following expression [5]:

$$
P=\frac{1}{2} \rho \cdot A \cdot C p(\lambda, \beta) \cdot V_{v}^{3}
$$

where:

- $P$ is the mechanic power produced by the turbine (W)

- $C p(\lambda, \beta)$ is the power factor which, in general, depends on specific speed $\lambda$ and the pitch angle $\beta$ (only for machines with variable blade angle). This is an adimensional magnitude.
- The air density, $\rho$, in $\mathrm{kg} / \mathrm{m}^{3}$, the value used commonly is $1.225 \mathrm{~kg} / \mathrm{m}^{3}$.

- $A$ is the swept area in $\mathrm{m}^{2}$.

- $v_{v}$ is the wind speed in $\mathrm{m} / \mathrm{s}$.

To this power it is necessary to take into account mechanical and electrical losses of the generator. In permanent regime this can be expressed like:

$$
P_{e}=C_{\text {torque }} \cdot \eta_{g} \cdot P_{\text {turbine }}
$$

where:

- $P_{e}$ is the electric power of the machine

- $C_{\text {torque }}$ is the torque coefficient, which considers mechanical losses in the transmission unit. A current value is 0.9 .

- $\eta_{g}$ is the electrical efficiency of the generator. A typical value is 0.95 .

- $P_{\text {turbine }}$ is the mechanical power harvest from the wind.

The global efficiency (electrical energy generated with respect to the potential energy transported by the wind) usually is next to $32 \%$.

\section{Simulation results}

In order to verify the operation of these models, a wind ramp was applied. The ramp grows up from $6 \mathrm{~m} / \mathrm{s}$ to 25 $\mathrm{m} / \mathrm{s}$.

In the Figure 4, the real power in the $0.69 \mathrm{kV}$ bus obtained by $\mathrm{PSS} / \mathrm{E}$ is shown. $\mathrm{PSS} / \mathrm{E}$ gives the electrical power generated by the machine in 100 MVA base. Furthermore, Figure 5 shows the result obtained by using PSCAD. From this comparison, both models show a good agreement with the power curve of the wind turbine. A difference between both models is found in the simulation starting, PSS/E begins from a stationary state while in PSCAD the machine starting is considered. In the next figures, a time-translation to change the origin is made, so the starting is avoided.

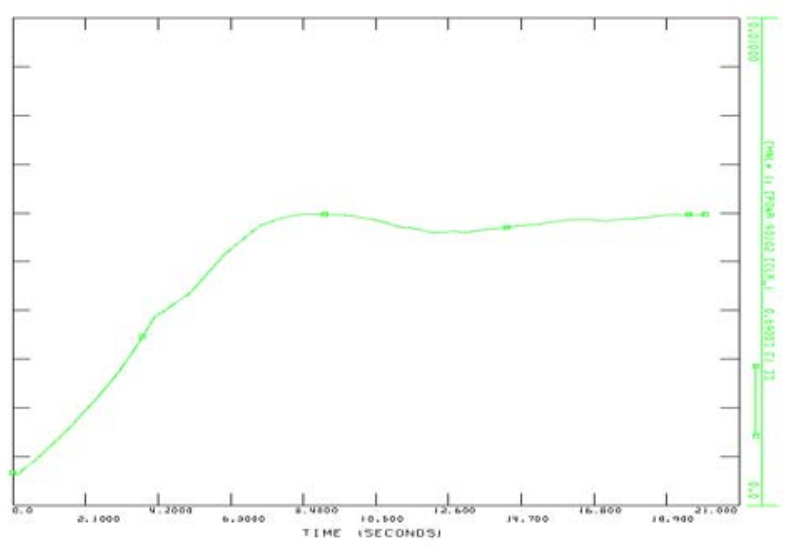

Figure 4. Real power obtained by the PSS/E model. 


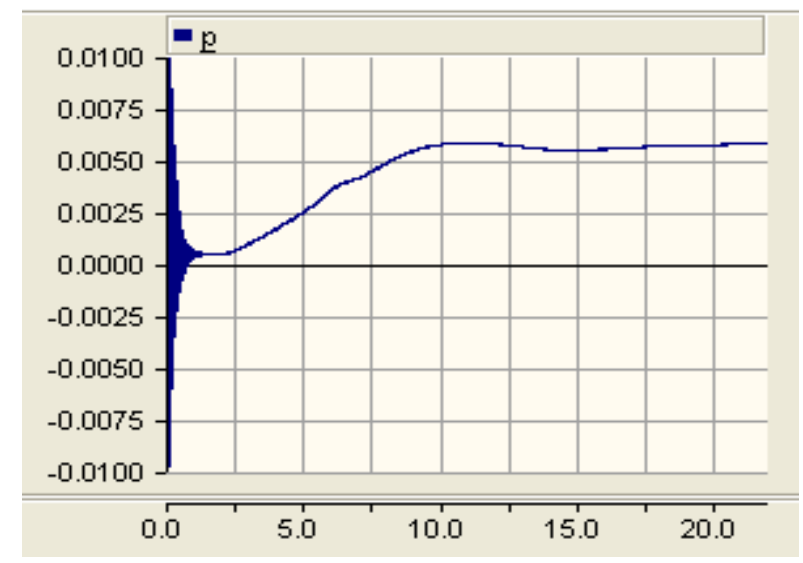

Figure 5. Real power obtained by the PSCAD model.

Figure 6 shows a comparison of the results obtained in an three-phase short circuit in the MV side of the transformer.

In Figure 7 a detail of this short circuit is given. The fault is applied in $\mathrm{t}=2 \mathrm{~s}$, and the duration is $200 \mathrm{~ms}$. In the PSCAD model, the power transient reaches 949,4 kVA, whereas in PSS/E model this transient is not observed.

Once the fault has been cleared, the recovering time is similar in both cases.

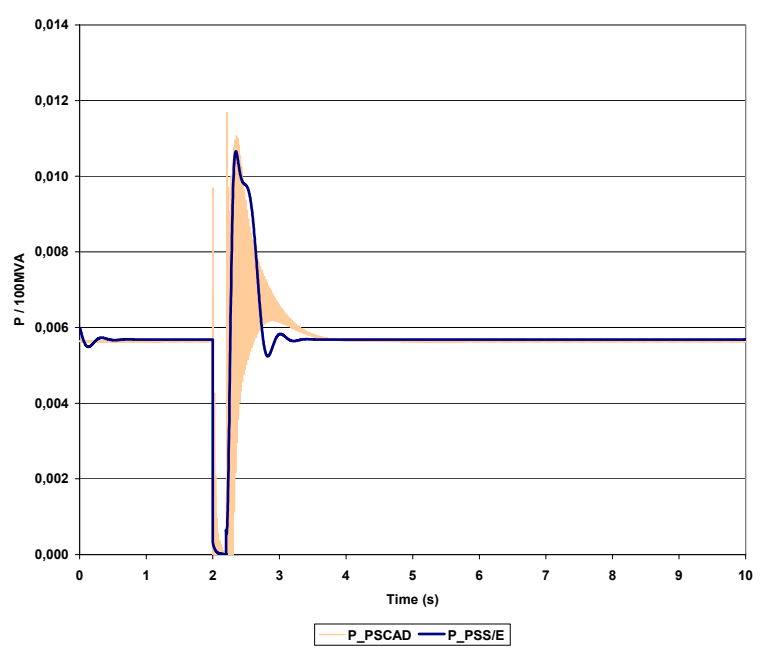

Figure 6. Three-phase short circuit.

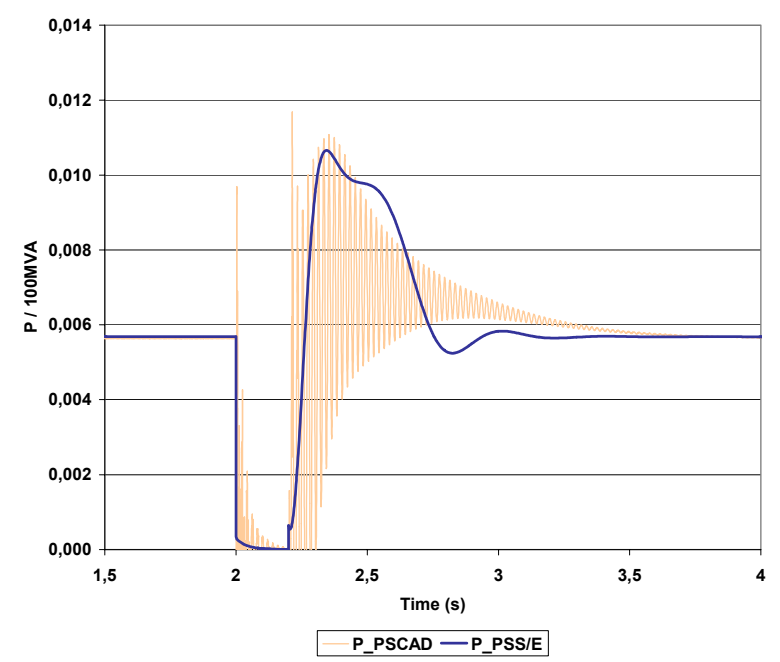

Figure 7. Detail of three-phase short circuit.

Furthermore, as it is shown in these figures, the PSS/E curve envelope to the PSCAD result.

\section{Conclusion}

A squirrel cage induction generator model developed in $\mathrm{PSS} / \mathrm{E}$ has been compared with an electromagnetic transient model (EMTDC). The results show as a dynamic tool as PSS/E cannot represent transient details. The dynamic curves obtained by using the PSS/E model envelope to the electromagnetic transient curves.

\section{References}

[1] PSS/E Power System Simulator for Engineering, Power Technologies Inc, version 26.2.3.

[2] Vladislav Akhmatov. "Analysis of Dynamic Behaviour of Electric Power System with Large Amount of Wind Power", $\mathrm{PhD}$ Thesis, Technical University of Denmark, 2003

[3] PSCAD / EMTDC V 3.0. Manitoba HVDC Research Centre Inc. 4th edition 2001

[4] Yao-Nan Yu, "Electric Power System Dynamics", Academic Press, 1983.

[5] A. E. Feijóo, "Influencia de los parques eólicos en la seguridad estacionaria y calidad de onda de redes eléctricas de gran dimensión", $\mathrm{PhD}$ Thesis, University of Vigo. 